\title{
Human amniotic epithelial cells inhibit growth of epithelial ovarian cancer cells via TGF-ß1-mediated cell cycle arrest
}

\author{
SHIXIA BU, QIUWAN ZHANG, QIAN WANG and DONGMEI LAI \\ The International Peace Maternity and Child Health Hospital, School of Medicine, \\ Shanghai Jiaotong University, Shanghai 200030, P.R. China
}

Received April 11, 2017; Accepted August 24, 2017

DOI: 10.3892/ijo.2017.4123

\begin{abstract}
It is reported that human amniotic epithelial cells (hAECs) endow intrinsic antitumor effects on certain kinds of cancer. This research was designed to evaluate whether hAECs endowed potential anticancer properties on epithelial ovarian cancer (EOC) cells in vivo and in vitro, which has not been reported before. In this study, we established a xenografted BALB/c nude mouse model by subcutaneously co-injecting ovarian cancer cell line, SK-OV-3, and hAECs for 28 days. In ex vivo experiments, CCK-8 cell viability assay, real-time PCR, cell counting assay, cell cycle analysis and immunohistochemistry (IHC) assay were used to detect the effects of hAEC-secreted factors on the proliferation and cell cycle progression of EOC cells. A cytokine array was conducted to detect anticancer-related cytokines released from hAECs. Human recombinant TGF- $\beta 1$ and TGF- $\beta 1$ antibody were used to treat EOC cells and analyzed whether TGF- $\beta 1$ contributed to the cell cycle arrest. Results from in vivo and ex vivo experiments showed that hAEC-secreted factors and rhTGF- $\beta 1$ decreased proliferation of EOC cells and induced G0/G1 cell cycle arrest in cancer cells, which could be partially
\end{abstract}

Correspondence to: Professor Dongmei Lai, The International Peace Maternity and Child Health Hospital, School of Medicine, Shanghai Jiaotong University, Shanghai 200030, P.R. China E-mail: laidongmei@hotmail.com

Abbreviations: hAECs, human amniotic epithelial cells; EOC, epithelial ovarian cancer; TGF- $\beta 1$, transforming growth factor- $\beta 1$; PCR, real-time polymerase chain reaction; IHC, immunohistochemistry; pRB, retinoblastoma protein; JNK, phospho-c-Jun N-terminal kinases; hAEC-CM, hAEC-conditioned medium; FBS, fetal bovine serum; PFA, paraformaldehyde; H\&E, hematoxylin and eosin; PCNA, proliferating cell nuclear antigen; GFP, green fluorescent protein; HRP, horseradish peroxidase; ANOVA, analysis of variance; $\mathrm{POI} / \mathrm{POF}$, premature ovarian failure and insufficiency; GM-CSF, granulocyte/macrophage colonystimulating factor; IL, interleukin; IFN- $\gamma$, interferon- $\gamma$; TNF- $\alpha$, tumor necrosis factor- $\alpha$; ELISA, enzyme-linked immunosorbent assay

Key words: human amniotic epithelial cell, epithelial ovarian cancer, cell cycle, TGF- $\beta 1$ reversed by excess TGF- $\beta 1$ antibody. These data indicate that hAECs endow potential anticancer properties on epithelial ovarian cancer in vivo and in vitro which is partially mediated by hAEC-secreted TGF- $\beta 1$-induced cell cycle arrest. This study suggests a potential application of hAEC-based therapy against epithelial ovarian cancer.

\section{Introduction}

The amniotic membrane is the innermost layer of fetal membrane that encloses the amniotic fluid and the fetus during pregnancy. Human amniotic epithelial cells (hAECs) can be easily obtained from amnion membrane with enzymatic digestion after being separated from chorion (1). hAECs are reported to have the ability to differentiate into all three germ layers in vitro (2). In addition, hAECs express markers of embryonic or germ cells (SSEA-3/4, TRA 1-60, and TRA 1-81), and some transcription factors of pluripotent stem cells (Oct-4, Sox-2, and Nanog) (3). Owning to their easy isolation, low-immunogenicity, anti-inflammatory properties, no tumorigenicity, and no ethical consideration, hAECs have gained increasing attention in regenerative medicine therapy, such as treating primary ovarian insufficiency (4), alveolar defect (5), skin regeneration (6) and so on.

Some unique traits of hAECs have attracted increasing attention about the potential anticancer properties of hAECs, such as induction apoptosis in lymphocytes and inhibition angiogenesis in a rat dorsal skinfold chamber model (7). Niknejad et al reported that hAEC-conditioned medium (hAEC-CM) could induce apoptosis in HeLa cells and MDA-MB-231 cells in vitro (8). Kang et al showed that hAECs displayed anticancer activity in a breast cancer-bearing nude mouse model through both cell-to-cell contact and paracrine way (9). However, Mamede et al revealed adverse effects of amniotic membrane-extracted proteins on human cancer cell lines tested by MTT assay in vitro (10).

The effects of hAECs on human epithelial ovarian cancer (EOC) have not been reported before. To characterize whether hAECs have innate antitumor effects on EOC cells in vivo, we established an SK-OV-3/hAECs co-injected xenografted BALB/c nude mouse model. In addition, we used hAEC-CM or Transwell co-culture system to detect the influences of hAEC-secreted factors on proliferation and cell cycle progression of EOC cells in vitro. Immunohistochemistry (IHC) 
was used to test the effects of hAECs on regulators of cell cycle progression, including p16 ${ }^{\mathrm{INK} 4 \mathrm{~A}}, \mathrm{p} 21$, phospho-c-Jun N-terminal kinases (JNK) and phospho-pRB (Ser807) (phospho-retinoblastoma protein) in tumor tissues. Cytokine array was conducted to discover anticancer-related cytokines released from hAECs, which showed that TGF- $\beta 1$ was the most abundant cell cycle-regulatory cytokine secreted from hAECs. Recombinant human TGF- $\beta 1$ (rhTGF- $\beta 1$ ) was used to treat EOC cells and then cellular viability and cell cycle were analyzed. TGF- $\beta 1$ antibody was added in the Transwell system to neutralize hAEC-secreted TGF- $\beta 1$ and then cell cycle of EOC cells was analyzed.

\section{Materials and methods}

Isolation and culture of human amniotic epithelial cells. Human placentas were obtained at term pregnancy during uncomplicated Caesarean sections with written and informed consent from women who were negative for HIV-I, and hepatitis B and C. This study has been approved by the Institutional Ethics Committee of the International Peace Maternity and Child Health Hospital, and written informed consent was obtained from all participants. The protocol of hAECs isolation was described previously (11). hAECs was cultured in DMEM/F12 (Thermo Fisher Scientific) medium supplemented with $10 \%$ fetal bovine serum (FBS; Thermo Fisher Scientific), streptomycin (100 U/ml; Thermo Fisher Scientific), penicillin (100 U/ml; Thermo Fisher Scientific), 1X non-essential amino acids (Thermo Fisher Scientific), and $1 \mathrm{mM}$ of sodium pyruvate (Thermo Fisher Scientific), and incubated at $37^{\circ} \mathrm{C}$ in an incubator containing $5 \% \mathrm{CO}_{2}$. Once the density of cells reached $80-90 \%$ confluency, cells were collected for subsequent experiments. hAECs $\left(5 \times 10^{6}\right)$ were seeded in $100 \mathrm{~mm}$ diameter dishes (Corning, USA) in complete culture medium for $72 \mathrm{~h}$. The hAEC-CM were then collected, $0.22 \mathrm{~mm}$ filtered, and used in subsequent experiments.

Immunofluorescence. hAECs were identified by using the indirect immunofluorescent labeling technique to detect the expression of CK-7 and vimentin. Cells were fixed with $4 \%$ paraformaldehyde (PFA), and then incubated with the following primary antibodies at $4{ }^{\circ} \mathrm{C}$ overnight: CK-7 (1:200 dilution; Boster, Wuhan, China) and vimentin (1:200 dilution; Boster). After that, cells were incubated with secondary antibody conjugated with Alexa Fluor ${ }^{\circledR} 488$ (1:200; Thermo Fisher Scientific). Cells were counterstained with DAPI (Thermo Fisher Scientific) and examined under the fluorescence microscope (Leica, Germany).

Cancer cell lines culture. The EOC cell lines, SK-OV-3 and A2780 were obtained from Shanghai Cell Bank of Chinese Academy of Sciences and cultured in DMEM/high glucose (Thermo Fisher Scientific) medium supplemented with $10 \%$ FBS, streptomycin $(100 \mathrm{U} / \mathrm{ml})$ and penicillin $(100 \mathrm{U} / \mathrm{ml})$. Cells were incubated at $37^{\circ} \mathrm{C}$ in an incubator containing $5 \% \mathrm{CO}_{2}$. Once the density of cells reached $80-90 \%$ confluency, cells were collected for subsequent experiments.

$C C K-8$ assay. Cell viability was detected with cell counting kit- 8 (CCK8; Dojindo Molecular Technologies, Japan) according to the manufacturer's protocol. EOC cells ( 7,000 cells per well) were seeded in 96-well plate with complete culture medium or hAEC-CM or mixture of complete culture medium and hAEC-CM at 1:1 ratio or different concentration of rhTGF- $\beta 1$ for $48 \mathrm{~h}$.

Co-culturing ovarian cancer cell lines and hAECs. Transwell co-culture system (Corning) was used to evaluate the effects of hAEC-secreted factors on EOC cells. hAECs $\left(1 \times 10^{6}\right.$ cells $)$ were seeded on Transwell inserts and EOC cells $\left(2 \times 10^{6}\right.$ cells $)$ were cultured in 6-well culture plates at the lower compartment. Cells were cultured in DMEM/high glucose medium supplemented with $10 \% \mathrm{FBS}$, streptomycin $(100 \mathrm{U} / \mathrm{ml})$ and penicillin $(100 \mathrm{U} / \mathrm{ml})$. All groups were incubated at $37^{\circ} \mathrm{C}$ in a $5 \% \mathrm{CO}_{2}$ incubator for 4 days.

RNA extraction and real-time polymerase chain reaction $(P C R)$. Total RNA was extracted from cultured EOC cells by TRIzol (Thermo Fisher Scientific) according to the manufacturer's instructions. Real-time quantitative PCR reactions were performed in triplicate, using the SYBR Green Real-time PCR Master Mix (Takara, Japan). PCR primers were designed according to cDNA sequences in the NCBI database. The following primers were used: GAPDH, 5'-AGGTCGGTGTG AACGGATTTG-3' and 5'-GGGGTCGTTGATGGCAACA-3'; PCNA, 5'-TTCCTGTGCAAAAGACGGAG-3' and 5'-TCACC GTTGAAGAGAGTGGA-3'; Ki-67, 5'-GAGGCAAATCA TCCGAACCC-3' and 5'-TTATTTTGGCGTCTGGAGCG-3'. Cycling conditions for the PCR machine were as follows: $95^{\circ} \mathrm{C}$ $5 \mathrm{sec}, 60^{\circ} \mathrm{C} 30 \mathrm{sec}$ and $72^{\circ} \mathrm{C} 30 \mathrm{sec}$ for 40 cycles. All reactions were performed in a $10-\mu 1$ volume. Gene expression levels were evaluated using the $\Delta \Delta \mathrm{Ct}$ method, standardized to levels of GAPDH amplification.

Establishment of xenografted nude mouse models. Twelve female BALB/c nude mice (4-week-old) were obtained from Shanghai Jiao Tong University School of Medicine (Shanghai, China). All experimental protocols were approved by the Ethics Committee of the School of Medicine of Shanghai Jiao Tong University, and were in accordance with the approved guidelines set by the Institutional Animal Care and Use Committee. The mice were housed under a laminar flow hood in an isolated room and were maintained under pathogen-free conditions. Five mice per group were subcutaneously injected with $2 \times 10^{6}$ SK-OV-3 or SK-OV-3/hAECs $\left(2 \times 10^{6}: 1 \times 10^{6}\right)$. Tumor volume was measured every two weeks and tumor weight was measured at the endpoint of the experiment. Animals were sacrificed by cervical dislocation under anesthetic status after 28 days. Tumor volume was calculated using the formula: tumor volume $\left(\mathrm{mm}^{3}\right)=0.52 \mathrm{x}$ [width $\left.\left(\mathrm{mm}^{2}\right)\right] \mathrm{x}$ [length (mm)] (12). To localize the hAECs in the tumor microenvironment, we transfected hAECs with green fluorescent protein (GFP) by lentivirus which was kindly provided by the Professor Lijian Hui (Chinese Academic of Sciences, Shanghai, China). Two mice were used to establish SK-OV-3/hAECs ${ }^{\text {GFP }}$ $\left(2 \times 10^{6}: 1 \times 10^{6}\right)$ xenografts and were sacrificed after 28 days.

Immunofluorescence (IF) staining and immunohistochemistry (IHC) staining. Tissue sections were deparaffinized and dehydrated. The slides were then incubated in a $3 \% \mathrm{H}_{2} \mathrm{O}_{2}$ solution 
A a

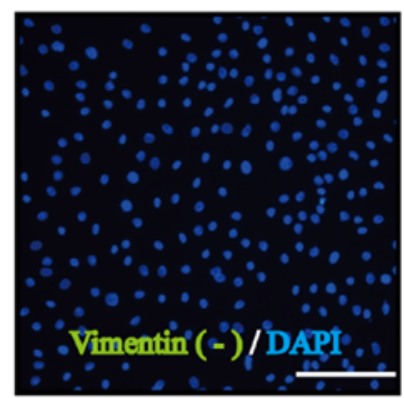

$\mathrm{B}$ a

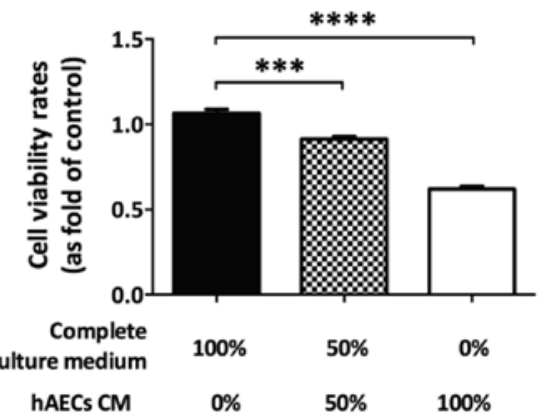

$\mathrm{C}_{\mathrm{a}}$

SK-OV-3

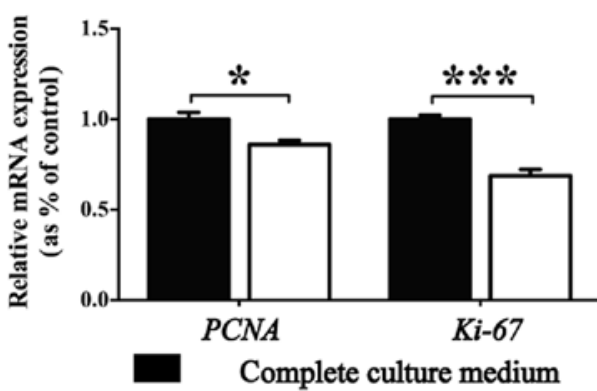

b

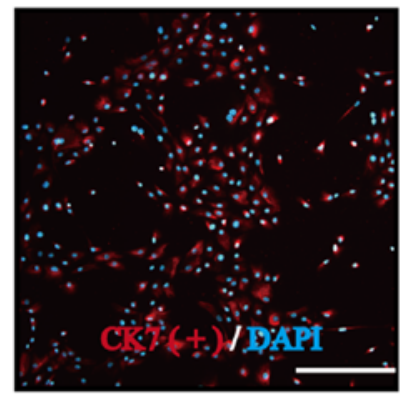

b

A2780

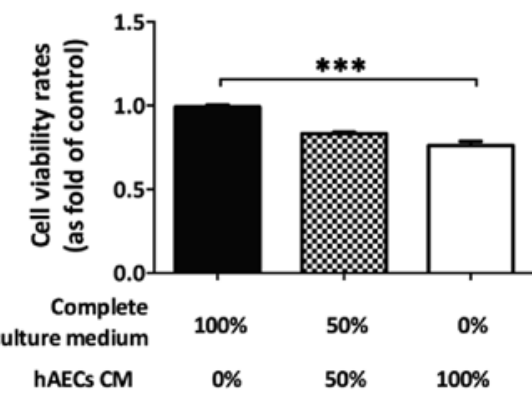

Figure 1. hAECs inhibit proliferation of EOC cells in vitro in a paracrine manner. (A) hAECs was negative for mesenchymal marker vimentin (green) and positive for epithelial marker CK-7 (red) by using immunofluorescence. DAPI (blue) staining showed the nuclei (scale bar, $100 \mu \mathrm{m}$ ). (B) CCK-8 cell viability assay used to test the effects of hAEC-CM in different concentrations on the viability of EOC cells at $48 \mathrm{~h}$ ( $\mathrm{n}=6$; performed in triplicate). Ordinary ANOVA was used for statistical analysis. (C) Real-time PCR were used to test the effects of hAECs on the expression levels of PCNA and Ki-67 in EOC cells cultured in Transwell system for $48 \mathrm{~h}\left(\mathrm{n}=3\right.$; performed in triplicate). Data are represented as means $\pm \mathrm{SEM}$. ${ }^{*} \mathrm{p}<0.05$ and ${ }^{* * *} \mathrm{p}<0.001$.

to block the endogenous peroxidase (IF staining can skip this procedure), followed by rinsing in PBS. To retrieve the antigenicity, sections were then treated with heated antigen retrieval solution containing ethylene diamine tetraacetic acid (EDTA; Wuhan Goodbio Technology, Wuhan, China). After being incubated with $5 \%$ bull serum albumin for 30 min to block the non-specific antibody binding sites, the samples were then incubated with the following primary antibodies at $4^{\circ} \mathrm{C}$ overnight: PCNA (1:6,000 dilution; CST, USA), Ki-67 (1:200 dilution; Arigo Biolaboratories, Taiwan, China), p16 ${ }^{\mathrm{INK} 4 \mathrm{~A}}$ (1:300 dilution; Wanleibio, China), p21 (1:300 dilution; Wanleibio), phospho-JNK (1:300 dilution; Wanleibio), phospho-pRB (Ser807) (1:500 dilution; Abcam, UK). For IHC analysis, horseradish peroxidase (HRP)-labelled anti-mouse/rabbit second antibodies and diaminobenzidine were used according to the manufacturer's instructions (Wuhan Goodbio Technology). Slides were counterstained with hematoxylin, differentiated with $0.1 \%$ hydrochloric acid alcohol, and washed by water.
They were then dehydrated through a graded ethanol series, immersed in xylene and mounted with permount ${ }^{\mathrm{TM}}$ mounting medium. For IF analysis for GFP, tissues were stained with secondary antibody conjugated with Alexa Fluor 488 (1:200; Thermo Fisher Scientific). Then tissues were counterstained with DAPI (Thermo Fisher Scientific) and examined under the fluorescence microscope (Leica).

For the semi-quantitative evaluation of $\mathrm{p} 21, \mathrm{p} 16^{\mathrm{INK} 4 \mathrm{~A}}$ and phospho-JNK expressions, we used a scoring method described by Budwit-Novotny et al (13). The evaluation of PCNA, Ki-67 and phospho-pRB (Ser807) were measured by the percentage of cells with positive signals in the nucleus.

Cytokine array. A human antibody array 1000 (a combination of human L-507 and human L-493) (RayBiotech, Inc., USA) was performed to detect the expression of anticancer-associated cytokines in hAECs. Samples of hAEC-CM were collected as previously described. Fresh DMEM/F12 culture medium 
A

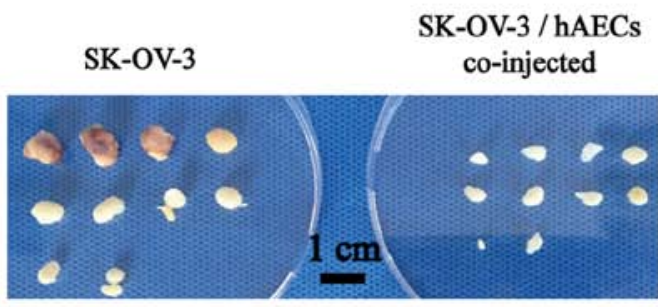

D a

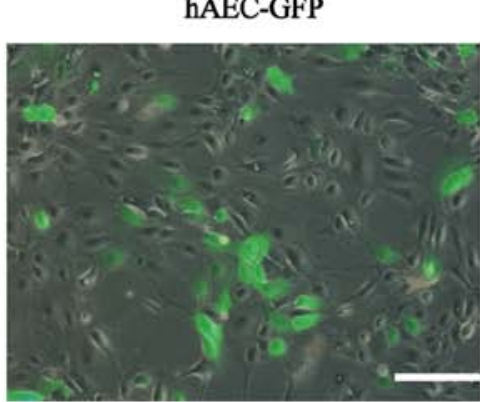

E a PCNA

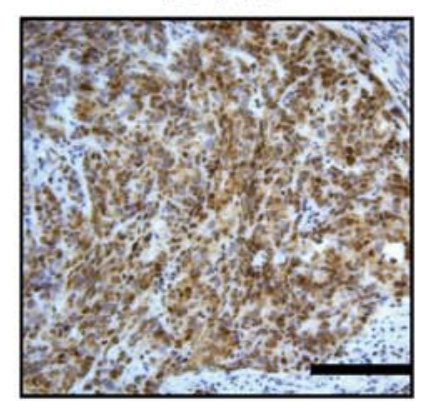

F a $\mathrm{Ki}-67$

SK-OV-3

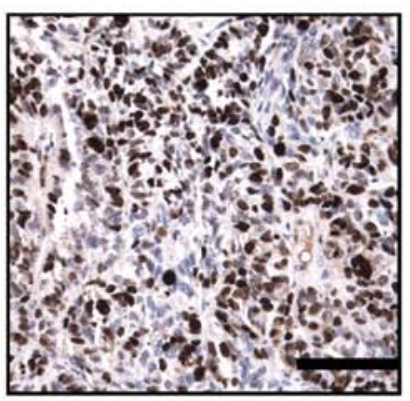

B

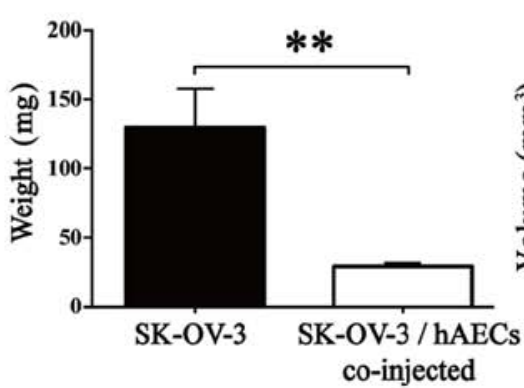

b

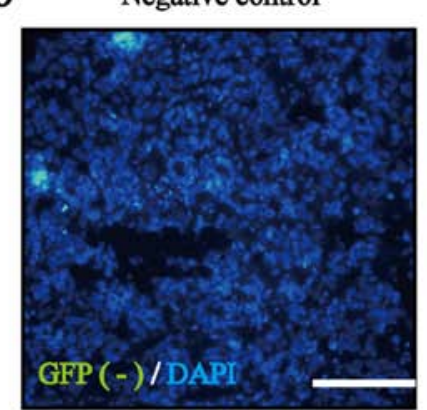

b

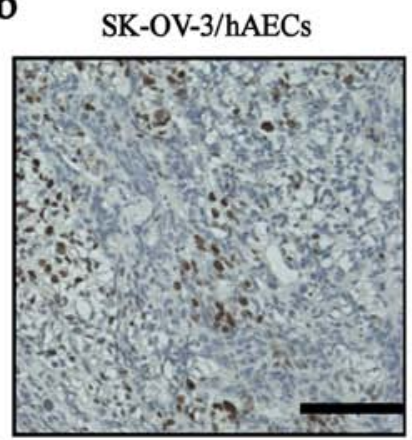

b

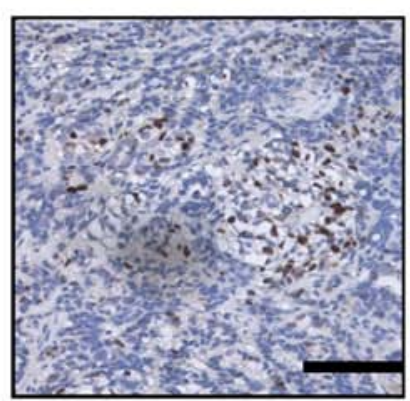

\section{c}

c

c
C SK-OV-3

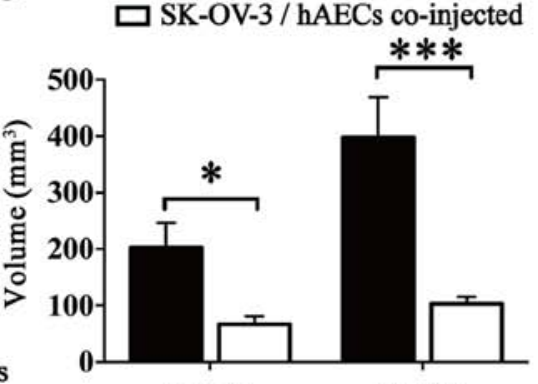

Day 14

SK-OV-3 / hAEC-GFP
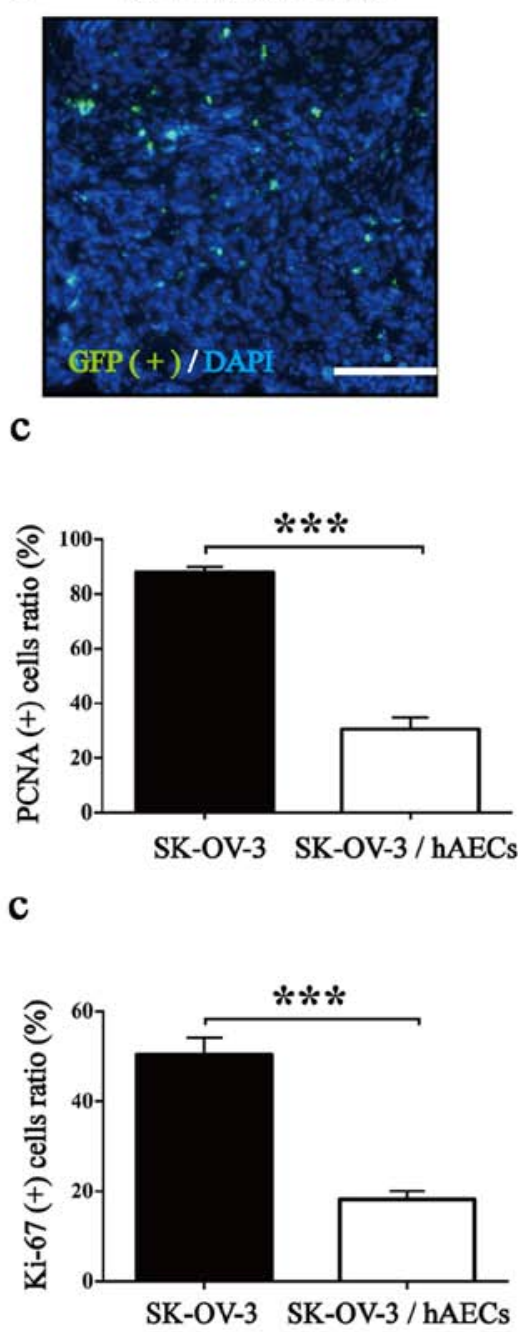

Figure 2. hAECs inhibit growth of SK-OV-3 cells in vivo. (A) Gross observation of subcutaneous xenografts obtained from SK-OV-3 injected group and SK-OV-3/hAECs co-injected group (scale bar, $1 \mathrm{~cm}$ ). (B) The average tumor weight of SK-OV-3 injected group was significantly higher than that of SK-OV-3/ hAECs co-injected group at day $28(\mathrm{n}=10)$. (C) The average tumor volume of SK-OV-3 injected group was significantly bigger than that of SK-OV-3/hAECs co-injected group at day 14 and day 28 (n=10). (D) (a) Representative image of GFP-transfected hAECs (scale bar, $200 \mu \mathrm{m}$ ). (b) We did not find positive signal of hAECs ${ }^{\mathrm{GFP}+}$ in the negative control (scale bar, $100 \mu \mathrm{m}$ ). (c) The positive signals were found in the xenografted tumor tissues obtained from SK-OV-3/ hAECs ${ }^{\mathrm{GFP}+}$ injected group (scale bar, $100 \mu \mathrm{m}$ ). (E and F) Proliferation of cancer cells were tested by IHC using antibodies against PCNA and Ki-67 in SK-OV-3 and SK-OV-3/hAECs tumor tissues (scale bar, $100 \mu \mathrm{m} ; \mathrm{n}=5$ ). Data were analyzed by Mann-Whitney U test and were represented as means \pm SEM. ${ }^{*} \mathrm{p}<0.05$, ${ }^{* *} \mathrm{p}<0.01$ and $^{* * *} \mathrm{p}<0.001$.

was used as the control. The cytokine array was performed according to the instructions. The intensities of signals were quantified by densitometry.

Cell cycle analysis. EOC cells were cultured alone or in the presence of hAEC-CM. After $48 \mathrm{~h}$, cancer cells were harvested by $0.25 \%$ trypsin/ethylene diamine tetraacetic acid, then being washed with phosphate-buffered solution, and fixed with $70 \%$ cold-ethanol for $24 \mathrm{~h}$ at $-20^{\circ} \mathrm{C}$. The cells were treated with $50 \mu \mathrm{g} / \mathrm{ml}$ RNase A (Tiangen, China) for $30 \mathrm{~min}$ at $37^{\circ} \mathrm{C}$ and then stained with $100 \mu \mathrm{g} / \mathrm{ml}$ propidium iodide (PI; Thermo Fisher Scientific) for $30 \mathrm{~min}$ at $4^{\circ} \mathrm{C}$. The cells 
$\mathrm{A}$ a
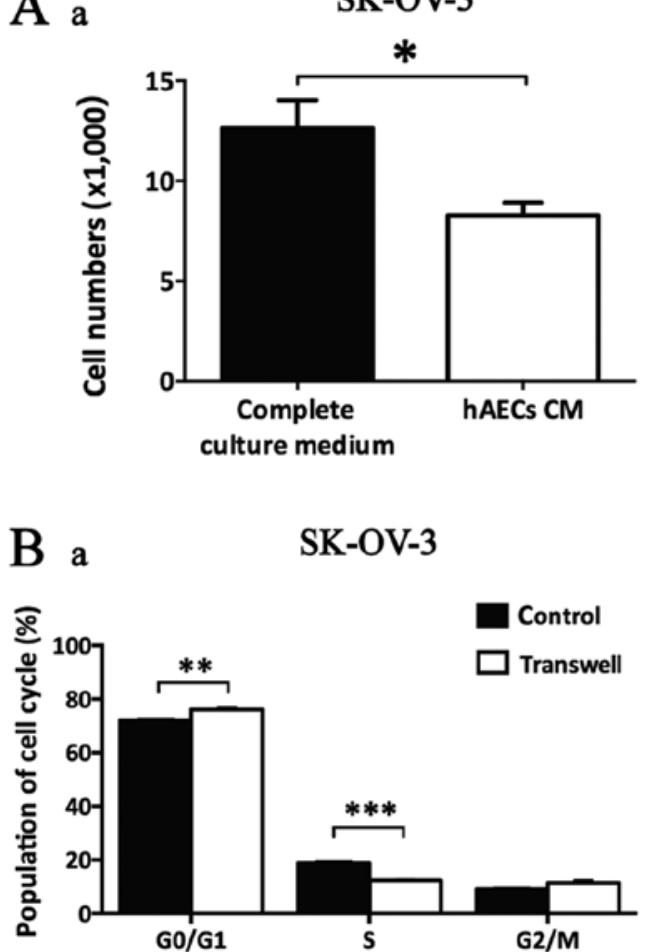

b

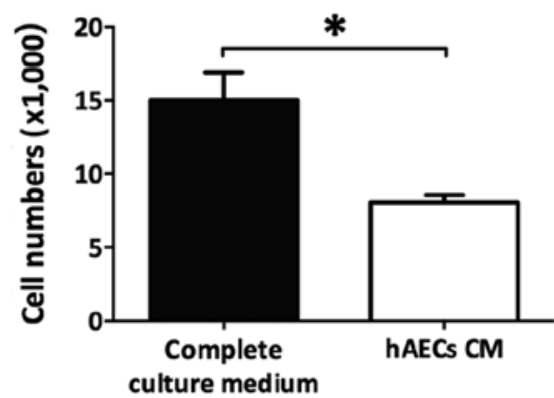

b

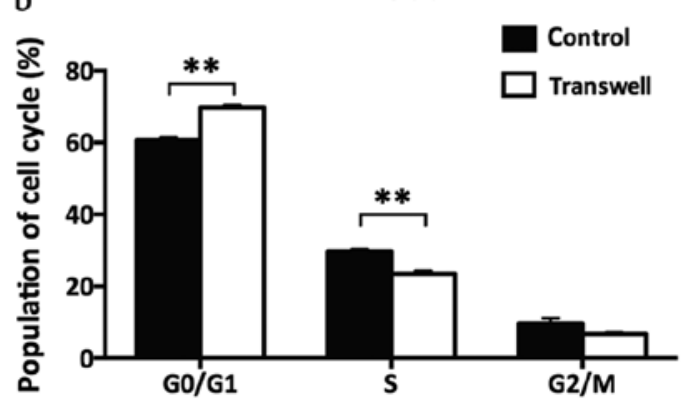

Figure 3. hAECs induce G0/G1 cell cycle arrest in EOC cells in a paracrine manner. (A) The effects of hAEC-CM on EOC cell division were tested by cell counting assay ( $\mathrm{n}=6$; performed in triplicate). (B) Cell cycle analysis revealed that hAEC-secreted factors induced G0/G1 cell cycle arrest in EOC cells in Transwell system ( $\mathrm{n}=3$; performed in triplicate). Data are represented as means $\pm \mathrm{SEM} .{ }^{*} \mathrm{p}<0.05,{ }^{* *} \mathrm{p}<0.01$ and ${ }^{* * *} \mathrm{p}<0.001$.

were analyzed using a Cytomics ${ }^{\mathrm{TM}}$ FC500 flow cytometer (Beckman Coulter, Brea, CA, USA). Data were analyzed using Beckman Coulter CXP software.

Enzyme-linked immunosorbent assay (ELISA). The ELISA kit was used to test the efficiency of TGF- $\beta 1$ antibody added to Transwell system to neutralize hAEC-secreted TGF- $\beta 1$ at $48 \mathrm{~h}$ (R\&D Systems, USA) and was conducted according to the manufacturer's instructions.

Statistical analysis. Results from three independent experiments are reported as the mean \pm SEM. Kolmogorov-Smirnov test was used to test whether data accorded with Gaussian distribution. Homogeneity of variance test was conducted by Bartlett's test. Two-tail Student's t-test or ordinary one-way analysis of variance (ANOVA) with Tukey's multiple comparisons test or Mann-Whitney U test (non-parametric test) or Kruskal-Wallis test (non-parametric test) was used to evaluate statistical differences via GraphPad Prism version 6 (GraphPad Software). Differences were considered significant at $\mathrm{p}$-value $<0.05$.

\section{Results}

hAECs inhibit proliferation of EOC cells in vitro in a paracrine manner. The hAECs represented a cobblestone-like morphology. These cells were positive for epithelial marker CK7, and negative for mesenchymal marker vimentin using IF analysis (Fig. 1A).

CCK-8 assay was conducted to test whether hAEC-secreted factors could influence the viability of EOC cells. Results showed that significant vitality inhibition in EOC cells was induced by hAEC-CM in different concentration compared with the control group at $48 \mathrm{~h}(\mathrm{n}=6)$. Moreover, the growth inhibition induced by hAEC-CM on EOC cells was significantly dose-dependent (Fig. 1B).

Results from real-time PCR showed that the expression levels of PCNA and Ki-67, two genes relating to cellular proliferation, were significantly downregulated within EOC cells co-cultured with hAECs compared with the control group (Fig. 1C; n=3).

hAECs inhibit growth of SK-OV-3 cells in a tumor-bearing nude mouse model. To explore the effects of hAECs on EOC cells in vivo, we established a tumor-bearing nude mouse model by subcutaneously co-injecting SK-OV-3 cells and hAECs at 2:1 ratio $\left(2 \times 10^{6}: 1 \times 10^{6}\right.$ cells; five mouse per group) at both sides of the scapular region. Four weeks after injection, we observed that hAECs significantly decreased the average weight and average volume of xenografted tumors compared with the control group (Fig. 2A-C; $n=10$ ). To further trace the location of hAECs in the tumor tissues, we labelled hAECs with GFP (Fig. 2D-a) and established a xenografted mouse model by subcutaneously co-injecting SK-OV-3 and hAEC ${ }^{\mathrm{GFP}+}$ cells at $2: 1$ ratio $\left(2 \times 10^{6}: 1 \times 10^{6}\right.$ cells; two mice were used) at both sides of the scapular region. After 28 days, hAEC ${ }^{\mathrm{GFP}+}$ cells were found in the stromal area of xenografted tumor tissues and no positive signal was observed in the negative control using IF assay (Fig. 2D-b and -c).

Results from IHC assay showed that hAECs significantly decreased the positive rates of both PCNA and Ki-67 in the SK-OV-3/hAECs co-injected tumor tissues compared with 


\section{A p16/INK4A}

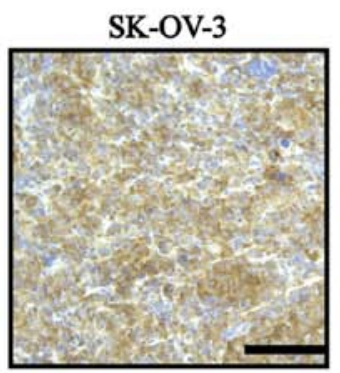

\section{B p 21}

SK-OV-3

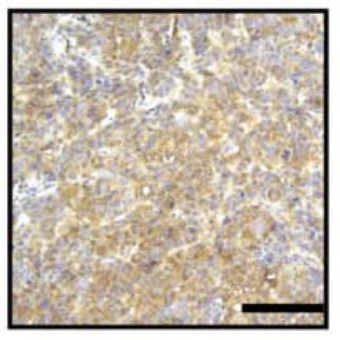

\section{C phospho-pRB (Ser807)}

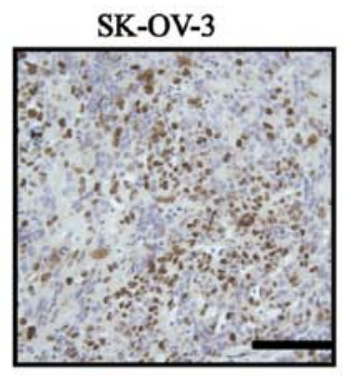

\section{D phospho-JNK}

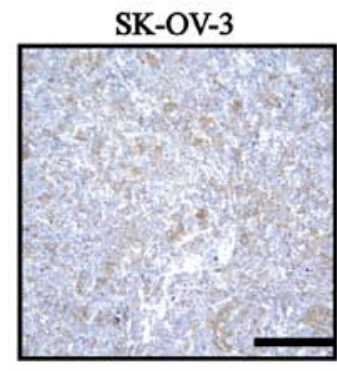

\section{SK-OV-3 / hAECs}

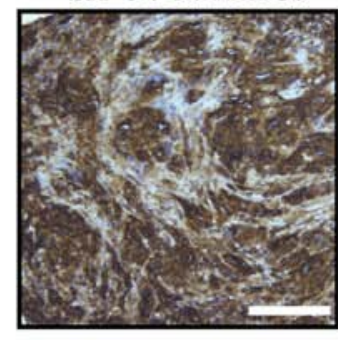

SK-OV-3 / hAECs
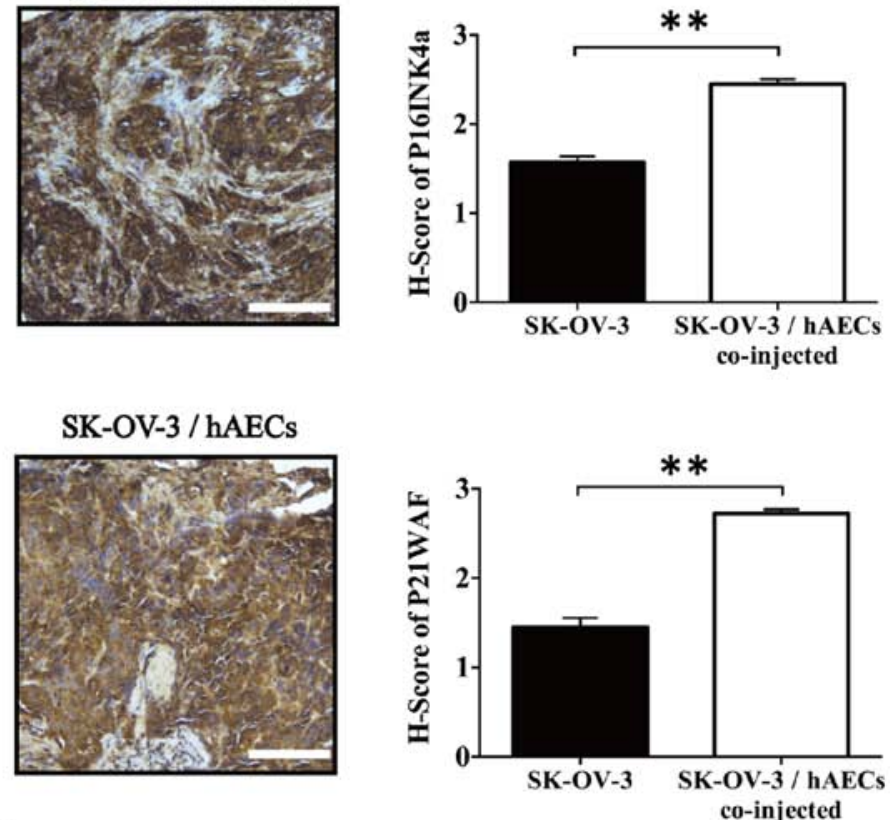
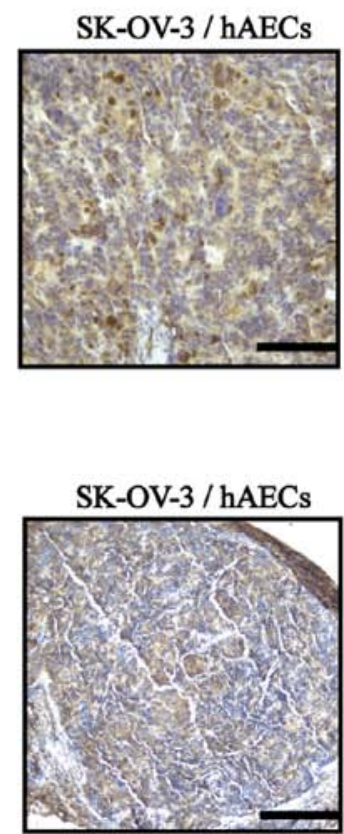
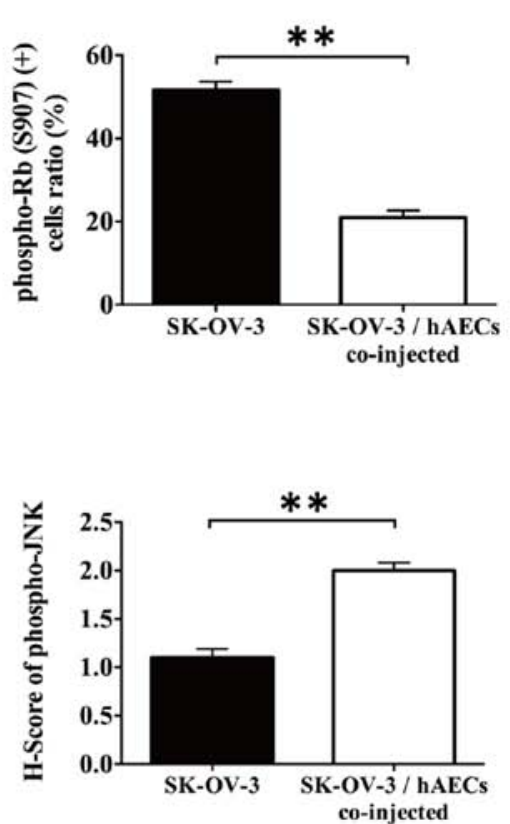

Figure 4. hAECs regulate the expression of proteins related to G0/G1 cell cycle arrest determined by IHC. hAECs upregulated p16 ${ }^{\mathrm{INK} 4 \mathrm{~A}}$, p21 and phospho-JNK expression and downregulated phospho-pRB (Ser807) expression in EOC cells (n=5). Scale bar of (A), (B) and (C) were $100 \mu \mathrm{m}$ and that of (D) was $50 \mu \mathrm{m}$. $\mathrm{H}$-score system was used for semi-quantification of $\mathrm{p} 16^{\mathrm{INK} 4 \mathrm{~A}}$, p21 and phospho-JNK between two groups. Data were analyzed by Mann-Whitney U test and presented as means \pm SEM. ${ }^{* *} \mathrm{p}<0.01$.

that in the only SK-OV-3 injected tumor tissues (Fig. 2E and F; $\mathrm{n}=5$ ), which was consistent with the ex vivo results.

hAECs induce G0/G1 cell cycle arrest in EOC cells in a paracrine manner. Cell count assay showed that hAEC-CM significantly decreased the cell numbers of both SK-OV-3 and A2780 cells at $48 \mathrm{~h}$, indicating hAEC-secreted factors influenced the division of EOC cells (Fig. 3A; $n=3$ ).

By using Transwell system, we observed that hAECs significantly induced G0/G1 cell cycle arrest in both SK-OV-3 and A2780 cells with a significant decrease in $\mathrm{S}$ phase using flow cytometry analysis (Fig. 3B; $n=3$ ).

hAECs influence expression of cell cycle-regulatory proteins in vivo. We further tested the effects of hAECs on the expression levels of three negative regulators of cell cycle progression, p16 ${ }^{\mathrm{INK} 4 \mathrm{~A}}$ and $\mathrm{p} 21$, and phospho-JNK, which was associated with TGF- $\beta 1$-induced growth inhibition, in the tumor tissues (14-16). IHC assay revealed that SK-OV-3/ hAECs co-injected tumor tissues exhibited significantly 
$\mathrm{A}$ a

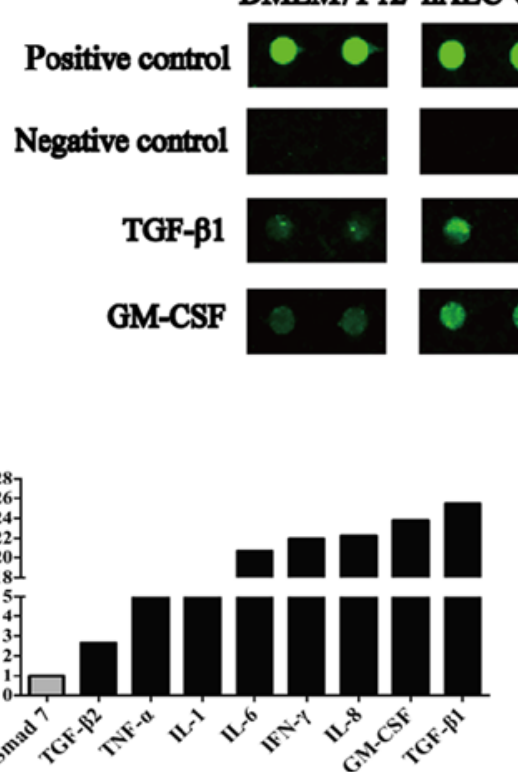

$\mathrm{C}_{\text {a }}$

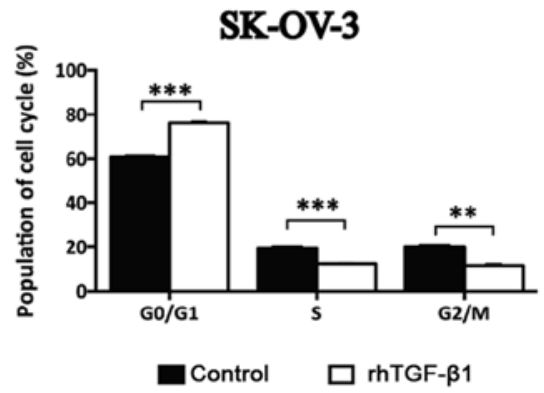

b

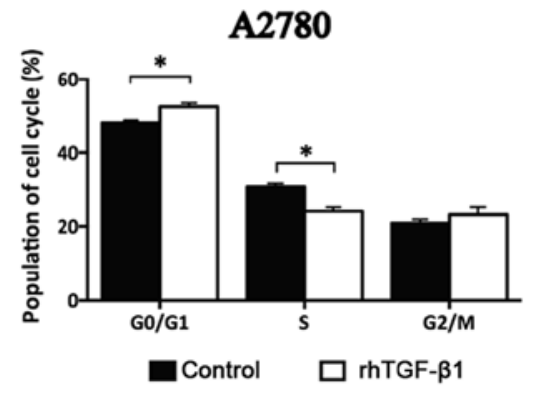

E a

SK-OV-3

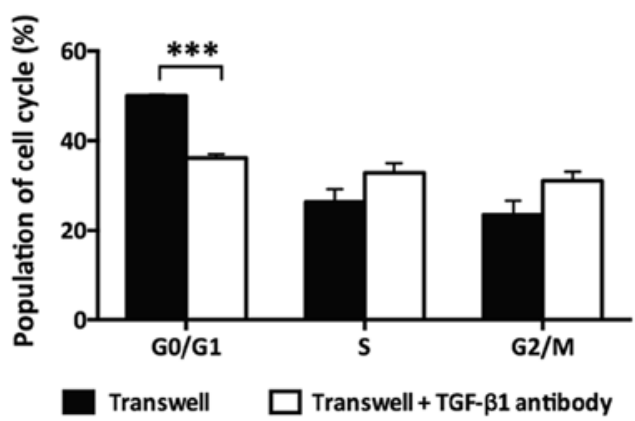

DMEM/F12 hAEC-CM

II-8

INF- $\gamma$

II-6

IL-1

B a

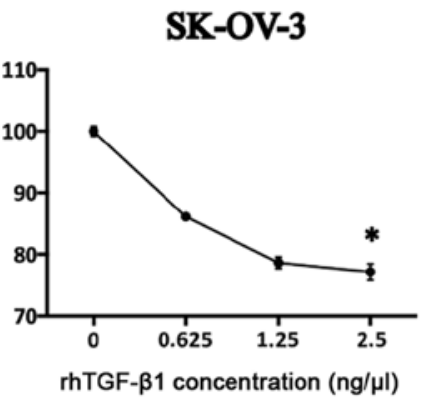

D

\section{DMEM/F12 hAEC-CM}

요 92
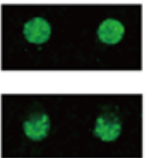

Smad 7

TGF- $\beta 2$
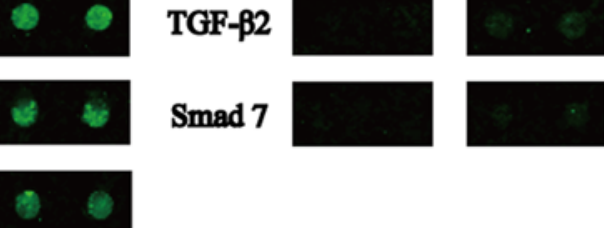
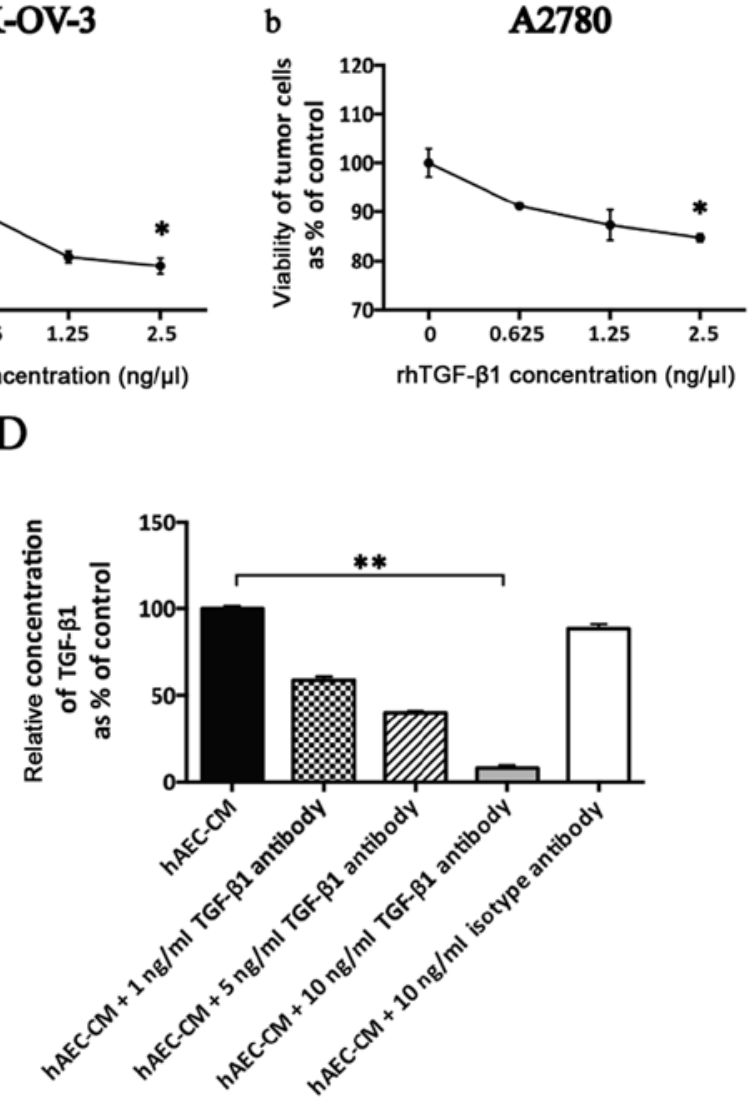

b

$\mathbf{A 2 7 8 0}$

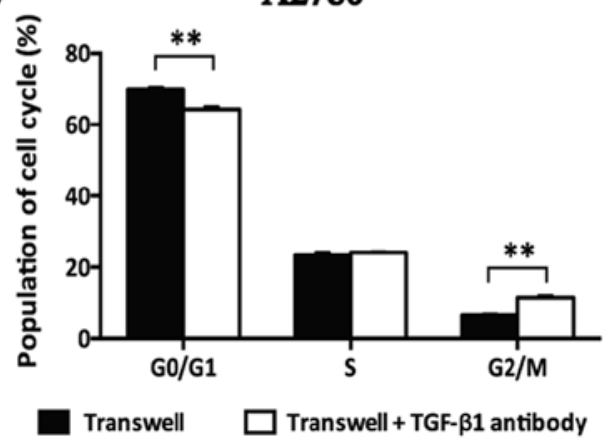

Figure 5. TGF- $\beta 1$ was enriched in hAEC-released cell cycle-regulatory cytokines and induced G0/G1 cell cycle arrest in EOC cells. (A) (a) Selective map of the human antibody array 1000. hAECs released multiple cell cycle-regulatory cytokines, including TGF- $\beta 1$, GM-CSF, IL-8, IFN- $\gamma$, IL-6, IL-1, TNF- $\alpha$, TGF- $\beta 2$ and Smad 7. (b) The intensities of the signals were quantified by densitometry and the expression of Smad 7 was regarded as control. (B) CCK-8 cell viability assay used to test the effects of rhTGF- $\beta 1$ in different concentrations on the viability of EOC cells at $48 \mathrm{~h}(\mathrm{n}=6$; performed in triplicate). Data were analyzed by Mann-Whitney U test. (C) After being treated with rhTGF- $\beta 1$, cell cycle of EOC cells was analyzed by flow cytometry. (D) ELISA was used to test the efficiency of TGF- $\beta 1$ antibody added to neutralize the function of hAEC-secreted TGF- $\beta 1$. Data were analyzed by Kruskal-Wallis test. (E) After being treated with TGF- $\beta 1$ antibody in the Transwell system, cell cycle of EOC cells was analyzed by flow cytometry. Data are presented as means \pm SEM. ${ }^{*}<0.05$, ${ }^{* *} \mathrm{p}<0.01$ and $^{* * *} \mathrm{p}<0.001$. 
higher expressions of p16 ${ }^{\mathrm{INK} 4 \mathrm{~A}}, \mathrm{p} 21$ and phospho-JNK compared with tumor tissues obtained from SK-OV-3 injected group (Fig. 4A-C; n=5). Furthermore, the level of phospho-pRB (Ser807), a major regulator controlling cell division cycle from $\mathrm{G} 1$ to $\mathrm{S}$ phase, was detected in the tumor tissues using IHC. Results showed that hAECs significantly inhibited phosphorylation of pRB (Ser807) within tumor tissues obtained from xenografts compared with control group (Fig. 4D; n=5).

TGF- $\beta 1$ is enriched in hAEC-released cell cycle-regulatory cytokines and induces $G 0 / G 1$ cell cycle arrest in EOC cells. The results from cytokine array revealed that hAECs express various anticancer-related cytokines, including TGF- $\beta 1$, granulocyte/macrophage colony-stimulating factor (GM-CSF), interleukin-8 (IL-8), IL-6, IL-1, interferon- $\gamma$ (IFN- $\gamma$ ), tumor necrosis factor- $\alpha$ (TNF- $\alpha$ ), TGF- $\beta 2$ and Smad 7 (Fig. 5A-a). The relative expression of cytokines was defined as the ratio of certain cytokine to Smad 7. We found that the level of hAEC-secreted TGF- $\beta 1$ was the most abundant among these cytokines which were reported to be able to negatively regulate cell cycle progression (Fig. 5A-b).

To illustrate the effects of TGF- $\beta 1$ on cell cycle progression within EOC cells, we conducted CCK-8 cell viability assay and found that the proliferative inhibition mediated by rhTGF- $\beta 1$ was dose-dependent (Fig. $5 \mathrm{~B}$; n=6). Further we added rhTGF- $\beta 1$ to treat EOC cells and then cell cycle progression was evaluated by flow cytometry. Results showed that rhTGF- $\beta 1$ induced a significant increase in G0/G1 phase and a decrease in $\mathrm{S}$ phase in both cell lines (Fig. 5C; $\mathrm{n}=3$ ).

To evaluate the neutralization efficiency of antibody used to attenuate the function of hAEC-secreted TGF- $\beta 1$, an ELISA kit was used. Results indicated that $10 \mathrm{ng} / \mathrm{ml}$ TGF- $\beta 1$ antibody showed the best efficiency of reducing the amount of hAEC-secreted TGF- $\beta 1$ in the supernatant (Fig. 5D).

Finally, we added TGF- $\beta 1$ antibody into the Transwell system and evaluated the cell cycle progression of EOC cells at $48 \mathrm{~h}$. We observed that TGF- $\beta 1$ antibody reversed hAEC-induced G0/G1 cell cycle arrest and increased the population in $\mathrm{S}$ phase and $\mathrm{G} 2 / \mathrm{M}$ phase (Fig. 5E; $\mathrm{n}=3$ ).

\section{Discussion}

hAECs show potent value in regenerative medicine. We have reported that both hAECs and hAEC-CM endowed ability of restoring fertility in mouse models of chemotherapy-induced POI/POF (premature ovarian failure and insufficiency) via inhibiting apoptosis in granular cells (11), transdifferentiating into granular cells (17), and enhancing angiogenesis (4). Before further clinical research and application, it is necessary to evaluate the safety of hAEC-based therapy in malignant situation, including the effects of hAECs on epithelial ovarian cancer which remains the leading cause of gynecological cancer-related death (18).

Many studies showed that hAECs might be a promising source for cell-based therapy for cancer, because of their ability to induce apoptosis (8) and stimulating cell cycle arrest (19). It is known that proliferation of cancer cells depends in part on cell cycle progression (20). hAEC-secreted factors have been shown to have the ability to induce cell cycle arrest in several cancer cells $(19,21)$. However, the specific factors and mechanisms responsible for the cell cycle-regulatory roles of hAECs on cancer cells still remain unclear. In this study, results from a cytokine array revealed that hAECs released various cytokines which are able to induce G0/G1 cell cycle arrest, including TGF- $\beta 1$ (22), GM-CSF (23), IL-8 (24), IL-6 (25), IL-1 (26), IFN- $\gamma$, TNF- $\alpha$ (27), TGF- $\beta 2$ (28) and Smad 7 (29). Our results showed that the secretion of TGF- $\beta 1$ derived from hAECs was abundant, this is consistent with the report of Kang et al showing the amount of TGF- $\beta 1$ ranked first among those anticancer-associated factors (9). We used recombinant human TGF- $\beta 1$ to imitate the function of hAEC-secreted TGF- $\beta 1$ on EOC cells. We observed that rhTGF- $\beta 1$ could inhibit the viability of EOC cells dose-dependently and induced G0/G1 cell cycle arrest in both cell lines. In addition, after confirming the concentration of TGF- $\beta 1$ antibody used to neutralize hAEC-secreted TGF- $\beta 1$ in the supernatant via ELISA, we found that hAEC-secreted factors-induced G0/G1 cell cycle arrest in Transwell system could be partially reversed by adding excess neutralizing TGF- $\beta 1$ antibody, indicating that hAEC-secreted TGF- $\beta 1$ play an important role in the induction of cell cycle arrest in EOC cells.

The cell cycle progression is well-organized by cyclindependent kinase (CDK) and CDK inhibitors. CDK activity is regulated by two families of inhibitors: INK4 proteins, including $\mathrm{p} 16^{\mathrm{INK} 4 \mathrm{~A}}, \mathrm{p} 15^{\mathrm{INK} 4 \mathrm{~B}}, \mathrm{p} 18^{\mathrm{INK} 4 \mathrm{C}}$ and $\mathrm{p} 19^{\mathrm{INK} 4 \mathrm{D}}$ and the Cip and Kip family, consisting of p21, p27 and p57 (30). Many studies reported that TGF- $\beta 1$ could increase the expression of p16 ${ }^{\mathrm{INK} 4 \mathrm{~A}}(14)$ and p21 (15), which functioned as negative regulators of cell cycle progression and might be associated with TGF- $\beta 1$-mediated G0/G1 cell cycle arrest. Schlosshauer et al reported that the loss of p16 ${ }^{\text {INK4A }}$ might attribute to tumor progression from ovarian borderline tumors to micropapillary tumors and low-grade carcinomas through overcoming the senescence barrier and/or indirect p53 downregulation (31). Kudoh et al suggested that deletion of $16^{\mathrm{INK} 4 \mathrm{~A}}$ and/or $\mathrm{p} 15^{\mathrm{INK} 4 \mathrm{~B}}$ was a potential indicator for poor chemotherapy response and adverse prognosis in ovarian cancer patients (32). Consistently, we found that lower expression of $\mathrm{p} 16^{\mathrm{INK} 4 \mathrm{~A}}$ was observed in larger xenografts obtained from SK-OV-3 injected group compared with tumor tissues obtained from SK-OV-3/hAECs co-injected group. In addition, our results showed that hAECs upregulated expression of p21 in tumor tissues and p21 overexpression was considered to indicate a better prognostic factor in epithelial ovarian cancer $(33,34)$. JNKs coordinate cell responses to stress and influence regulation of cell growth and transformation, whose phosphorylation is associated with TGF- $\beta 1$-induced growth inhibition (35) and protein stabilization of p21 (16). Herein our result was consistent with it and showed that hAECs upregulated level of phospho-JNK in xenografted model.

TGF- $\beta$ is a growth suppressing cytokine which maintains pocket proteins (p107, p130 and pRB) in an active, hypophosphorylated state through directly inducing expressing of CDK inhibitor proteins (36), including p16 ${ }^{\mathrm{INK} 4 \mathrm{~A}}$ and $\mathrm{p} 21$. By using a Rb1 knockout mouse model, Francis et al proved that hypophosphorylated $\mathrm{pRB}$ is required to inhibit expression of activator E2Fs (E2F1, E2F2 and E2F3) target genes, including positive regulators of cell cycle (PCNA and cyclin E1) (37). 
In this study, we found that hAECs decreased level of phospho-pRB (Ser807) in tumor tissues with lower expression of PCNA and higher levels of p16 ${ }^{\mathrm{INK} 4 \mathrm{~A}}, \mathrm{p} 21$ and phospho-JNK, indicating hAECs attenuated the malignant progression of EOC cells through halting cell cycle arrest in EOC cells.

In conclusion, we demonstrated the inhibitory effects of hAECs on epithelial ovarian cancer cells in vivo and in vitro. TGF- $\beta 1$ secreted from hAECs plays an important role in hAEC-mediated growth inhibition in epithelial ovarian cancer cells through inducing G0/G1 cell cycle arrest in cancer cells. This study provides a potential application of hAEC-based strategies against epithelial ovarian cancer. In addition, it suggests that hAEC-based therapy may be safe, but still needs further studies.

\section{Acknowledgements}

This study was supported by grants from National Natural ScienceFoundation of China(no.81370678),ShanghaiMunicipal Council for Science and Technology (no. 14411961500), Shanghai Municipal Education Commission-Gaofeng Clinical Medicine (no. 20152236), Shanghai Municipal Health Bureau (no. Y20140241), and Shanghai Jiao Tong University MedicineEngineering Fund (no. YG 2014QN12).

\section{References}

1. Barbati A, Grazia Mameli M, Sidoni A and Di Renzo GC: Amniotic membrane: Separation of amniotic mesoderm from amniotic epithelium and isolation of their respective mesenchymal stromal and epithelial cells. Curr Protoc Stem Cell Biol Chapter 1: 8, 2012.

2. Ilancheran S, Michalska A, Peh G, Wallace EM, Pera M and Manuelpillai U: Stem cells derived from human fetal membranes display multilineage differentiation potential. Biol Reprod 77: 577-588, 2007.

3. Miki T, Lehmann T, Cai H, Stolz DB and Strom SC: Stem cell characteristics of amniotic epithelial cells. Stem Cells 23: 1549-1559, 2005

4. Yao X, Guo Y, Wang Q, Xu M, Zhang Q, Li T and Lai D: The paracrine effect of transplanted human amniotic epithelial cells on ovarian function improvement in a mouse model of chemotherapy-induced primary ovarian insufficiency. Stem Cells Int 2016: 4148923, 2016.

5. Si JW, Zhang JJ, Dai JW, Yu DD, Yu HB, Shi J, Wang XD, Shen SGF and Guo LH: Osteogenic differentiation of human amniotic epithelial cells and its application in alveolar defect restoration. Stem Cells Transl Med 3: 1504-1513, 2014.

6. Jiang LW, Chen $\mathrm{H}$ and $\mathrm{Lu} \mathrm{H}$ : Using human epithelial amnion cells in human de-epidermized dermis for skin regeneration. J Dermatol Sci 81: 26-34, 2016.

7. Niknejad H, Paeini-Vayghan G, Tehrani FA, Khayat-Khoei M and Peirovi H: Side dependent effects of the human amnion on angiogenesis. Placenta 34: 340-345, 2013.

8. Niknejad H, Khayat-Khoei M, Peirovi $\mathrm{H}$ and Abolghasemi H: Human amniotic epithelial cells induce apoptosis of cancer cells: A new anti-tumor therapeutic strategy. Cytotherapy 16: 33-40, 2014.

9. Kang NH, Yi BR, Lim SY, Hwang KA, Baek YS, Kang KS and Choi KC: Human amniotic membrane-derived epithelial stem cells display anticancer activity in BALB/c female nude mice bearing disseminated breast cancer xenografts. Int J Oncol 40 : 2022-2028, 2012.

10. Mamede AC, Laranjo M, Carvalho MJ, Abrantes AM, Pires AS Brito AF, Moura P, Maia CJ and Botelho MF: Effect of amniotic membrane proteins in human cancer cell lines: An exploratory study. J Membr Biol 247: 357-360, 2014.

11. Zhang Q, Xu M, Yao X, Li T, Wang Q and Lai D: Human amniotic epithelial cells inhibit granulosa cell apoptosis induced by chemotherapy and restore the fertility. Stem Cell Res Ther 6: $152,2015$.
12. Liu T, Zhao L, Zhang Y, Chen W, Liu D, Hou H, Ding L and Li X: Ginsenoside 20(S)-Rg3 targets HIF-1 $\alpha$ to block hypoxia-induced epithelial-mesenchymal transition in ovarian cancer cells. PLoS One 9: e103887, 2014.

13. Budwit-Novotny DA, McCarty KS, Cox EB, Soper JT, Mutch DG, Creasman WT, Flowers JL and McCarty KS Jr: Immunohistochemical analyses of estrogen receptor in endometrial adenocarcinoma using a monoclonal antibody. Cancer Res 46: 5419-5425, 1986.

14. Jin G, Cao Z, Sun X, Wang K, Huang T and Shen B: Protein O-glucosyltransferase 1 overexpression downregulates p16 in BT474 human breast cancer cells. Oncol Lett 8: 594-600, 2014.

15. Datto MB, Yu Y and Wang XF: Functional analysis of the transforming growth factor beta responsive elements in the WAF1/Cip1/p21 promoter. J Biol Chem 270: 28623-28628, 1995.

16. Kim GY, Mercer SE, Ewton DZ, Yan Z, Jin K and Friedman E: The stress-activated protein kinases $\mathrm{p} 38$ alpha and JNK1 stabilize p21(Cip1) by phosphorylation. J Biol Chem 277: 29792-29802, 2002.

17. Wang F, Wang L, Yao X, Lai D and Guo L: Human amniotic epithelial cells can differentiate into granulosa cells and restore folliculogenesis in a mouse model of chemotherapy-induced premature ovarian failure. Stem Cell Res Ther 4: 124, 2013.

18. Jayson GC, Kohn EC, Kitchener HC and Ledermann JA: Ovarian cancer. Lancet 384: 1376-1388, 2014.

19. Di Germanio C, Bernier M, Petr M, Mattioli M, Barboni B and de Cabo R: Conditioned medium derived from rat amniotic epithelial cells confers protection against inflammation, cancer, and senescence. Oncotarget 7: 39051-39064, 2016.

20. Dickson MA: Molecular pathways: CDK4 inhibitors for cancer therapy. Clin Cancer Res 20: 3379-3383, 2014.

21. Magatti M, De Munari S, Vertua E and Parolini O: Amniotic membrane-derived cells inhibit proliferation of cancer cell lines by inducing cell cycle arrest. J Cell Mol Med 16: 2208-2218, 2012.

22. Chen X, Zheng S, Gao Y, Dai H, Mou H and Yang H: Growth regulation of ovarian cancer cell line HO-8910 by transforming growth factor beta 1 in vitro. Chin Med J (Engl) 111: 546-550, 1998.

23. Ketley NJ, Allen PD, Kelsey SM and Newland AC: Modulation of idarubicin-induced apoptosis in human acute myeloid leukemia blasts by all-trans retinoic acid, 1,25(OH)2 vitamin D3, and granulocyte-macrophage colony-stimulating factor. Blood 90: 4578-4587, 1997.

24. Schinke C, Giricz O, Li W, Shastri A, Gordon S, Barreyro L, Bhagat T, Bhattacharyya S, Ramachandra N, Bartenstein M, et al: IL8-CXCR2 pathway inhibition as a therapeutic strategy against MDS and AML stem cells. Blood 125: 3144-3152, 2015.

25. Resnitzky D, Tiefenbrun N, Berissi H and Kimchi A: Interferons and interleukin 6 suppress phosphorylation of the retinoblastoma protein in growth-sensitive hematopoietic cells. Proc Natl Acad Sci USA 89: 402-406, 1992.

26. Muthukkumar S, Sells SF, Crist SA and Rangnekar VM: Interleukin-1 induces growth arrest by hypophosphorylation of the retinoblastoma susceptibility gene product RB. J Biol Chem 271: 5733-5740, 1996.

27. Lillibridge $\mathrm{CD}$ and $\mathrm{O}^{\prime}$ Connell $\mathrm{BC}$ : In human salivary gland cells, overexpression of E2F1 overcomes an interferon-gamma- and tumor necrosis factor-alpha-induced growth arrest but does not result in complete mitosis. J Cell Physiol 172: 343-350, 1997.

28. Wu DT, Bitzer M, Ju W, Mundel P and Böttinger EP: TGF-beta concentration specifies differential signaling profiles of growth arrest/differentiation and apoptosis in podocytes. J Am Soc Nephrol 16: 3211-3221, 2005.

29. Kitamura K, Aota S, Sakamoto R, Emori T and Okazaki K: Smad7 induces G0/G1 cell cycle arrest in mesenchymal cells by inhibiting the expression of G1 cyclins. Dev Growth Differ 47: 537-552, 2005.

30. Malumbres M and Barbacid M: Cell cycle, CDKs and cancer: A changing paradigm. Nat Rev Cancer 9: 153-166, 2009.

31. Schlosshauer PW, Deligdisch L, Penault-Llorca F, Fatemi D, Qiao R, Yao S, Pearl M, Yang Z, Sheng T and Dong J: Loss of p16INK4A expression in low-grade ovarian serous carcinomas. Int J Gynecol Pathol 30: 22-29, 2011.

32. Kudoh K, Ichikawa Y, Yoshida S, Hirai M, Kikuchi Y, Nagata I, Miwa M and Uchida K: Inactivation of p16/CDKN2 and p15/ MTS2 is associated with prognosis and response to chemotherapy in ovarian cancer. Int J Cancer 99: 579-582, 2002. 
33. Buchynska LG, Nesina IP, Yurchenko NP, Bilyk OO Grinkevych VN and Svintitsky VS: Expression of p53, p21WAF1/ CIP1, p16INK4A and Ki-67 proteins in serous ovarian tumors. Exp Oncol 29: 49-53, 2007.

34. Bali A, O'Brien PM, Edwards LS, Sutherland RL, Hacker NF and Henshall SM: Cyclin D1, p53, and p21Waf1/Cip1 expression is predictive of poor clinical outcome in serous epithelial ovarian cancer. Clin Cancer Res 10: 5168-5177, 2004

35. Khalil N, Xu YD, O'Connor R and Duronio V: Proliferation of pulmonary interstitial fibroblasts is mediated by transforming growth factor-beta1-induced release of extracellular fibroblast growth factor- 2 and phosphorylation of p38 MAPK and JNK. J Biol Chem 280: 43000-43009, 2005.
36. Henley SA and Dick FA: The retinoblastoma family of proteins and their regulatory functions in the mammalian cell division cycle. Cell Div 7: 10, 2012.

37. Francis SM, Bergsied J, Isaac CE, Coschi CH, Martens AL, Hojilla CV, Chakrabarti S, Dimattia GE, Khoka R, Wang JY, et al: A functional connection between $\mathrm{pRB}$ and transforming growth factor beta in growth inhibition and mammary gland development. Mol Cell Biol 29: 4455-4466, 2009. 